Warszawskie Studia Pastoralne UKSW

Rok XI 2016 Nr 4(33)

Ks. Ireneusz Celary

\title{
JUNGE LEUTE UND KIRCHE, KIRCHE UND JUNGE LEUTE. EIN WECHSELSEITIG PROBLEMATISCH GEWORDENES VERHÄLTNIS. EINE PASTORALTHEOLOGISCHE REFLEXION
}

Die Kontakte zwischen den jungen Leuten und Kirche befinden sich heutzutage in einer kritischen Phase. Mit denjenigen oder gleichen Slogans verängstigt derzeit die Öffentlichkeit viele Gesellschaftswissenschaftler und Pressevertreter ${ }^{1}$. Nach ihrer Meinung haben Teenager gegenwärtig mit der Pfarrgemeinde wenig zu tun, weil ihr Lebensstil zeitgemäß und deshalb areligiös ist ${ }^{2}$. Ihre Aussagen sind zweifellos subjektiv, denn das Jugendalter ist sicherlich höchst variabel. Außerdem gibt es tatsächlich junge Erwachsene, die sich in Pfarrgemeinden ansammeln oder auch in solche flüchten. Es sollte jedoch betont werden, dass zurzeit das Christentum und die Kirche

1 Vgl. H. Barz, Religion ohne Institution? Eine Bilanz der sozialwissenschaftlichen Jugendforschung, Opladen 1992, S. 25-30; R. Inglehart, Kultureller Umbruch, Frankfurt-New York 1989, S. 245ff.

2 Vgl. S. Knobloch, Gottesleere? Wider die Rede vom Verlust des Göttlichen, Ostfildern 2013, S. 10; N. Possmann, Jugendpastoral, in: Lexikon des Apostolats. Stichworte verantworteten Glaubens, Hrsg. F. Courth, Limburg 1995, S. 154 -156; R. Sauer, Die Kunst, Gott zu feiern. Liturgie wiederentdecken und einüben, München 1996, S. 15--17; J. Zinnecker, Jugend, Kirche und Religion. Aktuelle empirische Ergebniss und Entwicklungstendenzen, in: Religionsunterricht im Abseits? Das Spannungsfeld Jugend, Schule, Religion, Hrsg. G. Hilger, G. Reilly, München 1993, S. 114-119. 
bis in die direkten Standpunkte hinein ihre Zuverlässigkeit eingebüßt haben ${ }^{3}$.

Am ausführlichsten erfahren wir das in der Pfarrgemeindearbeit. Dies führt zur Tatsache, dass ein großer Prozentsatz der Kirchengemeinden mit der Jugendseelsorge zurücktritt, da sie damit gescheitert sind $^{4}$. Mehr Aufmerksamkeit legt man manchmal in der Arbeit mit den alten Menschen, beziehungsweise auf kleine Gemeinschaften, die weniger belastend sind. Insbesondere entwickelt sich die Altenseelsorge, die ja ebenfalls in der Tat bedeutend ist ${ }^{5}$. In diesem Fall stellt sich aber die Frage, wie erscheint dann die Zukunft den Pfarrgemeinden? Ausgerechnet die Besorgnis um die Jugendlichen müsste, nach Hans-Joachim Höhn, eine privilegierte Aufgabe der Kirche sein ${ }^{6}$. Will man die Pfarrgemeinden erneut für sie „attraktiv“ machen, muss man sich eher informieren, warum sich Teenager dort immer weniger wie zu Hause fühlen und wie die Pfarrseelsorge in der Zukunft sein müsste, um das Zutrauen junger Menschen wiederzuerlangen ${ }^{7}$.

3 Vgl. B. Kranemann, Die Feier der christlichen Initiation, in: Heil erfahren in den Sakramenten, H. Hoping, B. Kranemann, S. Wahle, N. Weidinger, Freiburg-Basel-Wien 2009, S. 75-76; P. M. Zulehner, Kirche umbauen - nicht totsparen, Kevelaer 2009, S. 53-54; L. Karer, Handbuch der praktischen Gemeindearbeit, Freiburg-Basel-Wien 1990, S. 77-78.

4 Vgl. H. Wustmans, Firmung oder die Frage nach der Autorität der Kirche im Erleben Jugendlicher, in: „Theologisch-praktische Quartalschrift“158(2010), S. 371380; G. Schmidtchen, Gottesdienst in einer rationalen Welt, Stuttgart-Freiburg 1973, S. 166-167.

5 Vgl. E. Trapp, Seelsorge - eine (andere) Pflegequalität?, in: „Pastoralblatt“ 10(2011), S. 300-306; D. Nauer, Seelsorge. Sorge um die Seele, Stuttgart 2010, S. $202 \mathrm{ff}$.

6 Vgl. H. J. Höhn, Fremde Heimat Kirche. Glauben in der Welt von heute, Freiburg-Basel-Wien 2012, S. 117; A. Wollbold, Handbuch der Gemeinde-Pastoral, Regensburg 2004, S. 288; M. N. Ebertz, Die sieben »Jugendtabus « der Kirche. Soziologische Aspekte eines problematisch gewordenen Verhältnisses, in: „Katechetische Blätter“ 124(1999), S. 406-410.

7 Vgl. P. M. Zulehner, W. Müller, B. Sieberer (Hrsg.), Der Reichtum der Kirche sind ihre Menschen. Pfarrgemeinderäte beleben die Kirchengemeinden. Bericht über eine Umfrage, Ostfildern 2010, S. 50-51; R. Bleistein, Jugendpastoral - Entwurfeiner Neukonzeption, in: „Katechetische Blätter“100(1975), S. 69; L. Karer, Handbuch..., S. 117-118. 


\section{Junge Menschen fühlen sich in den Kirchen zurzeit nicht mehr wie „zu Hause“}

Als Reaktion auf diese Aussage sollte, nach Andreas Wollbold, zuerst betont werden, dass heutzutage Teenager und Kirchen sehr oft verschiedene Anschauungen haben, diese bringen sie nicht zum gemeinsamen Konsens ${ }^{8}$. Nach Ansicht vieler Seelsorger müssen Jugendliche für das Gemeindeleben mehr oder weniger eine „Eintrittskarte“, eine Legitimation, mitbringen, die annähernd Folgendes erweisen sollte: möglichst systematische Teilnahme an den Andachten; Absicht zu unterschiedlichen Hilfen in der Gemeinde: in Liturgie, im freundlichen Pfarrleben, im Ehrenamt (junge Menschen sollen zum „Herzeigen“ da sein); entsprechende Bedürfnisse an der Lebensweise: was die Bekleidung betrifft, die Freundlichkeit, der Umgang mit dem anderen Geschlecht bei anfangender Partnersuche usw ${ }^{9}$.

Die Heranwachsenden werden auch sehr oft nach den Qualitäten der ranghöheren Generation geschätzt. Bestätigt die Jugend diese nicht, heißt es, dass sie einfach keine Werte mehr haben, ohne sich $\mathrm{zu}$ informieren, ob sie nicht nur einen unterschiedlichen Maßstab hat. Manche Seelsorger erwarten auch von den Jugendlichen, dass sie herangereifte Christen seien. Die Forderungen an sie sind oft größer als jene an die restlichen Pfarrangehörigen oder man mutet ihnen nichts mehr zu, lässt sie spielen und jammert nur, wie anders die jungen Leute heutzutage sind und wie ehemals alle besser war ${ }^{10}$.

${ }^{8}$ Vgl. R. Bleistein, Jugend. Theologisch-pastoral, in: Lexikon der Pastoral, Hrsg. K. Baumgartner, P. Scheuchenpflug, Bd. 1 A-Ki, Freiburg-Basel-Wien 2002, S. 785786; H. J. Höhn, Fremde Heimat Kirche..., S. 121-122; F. J. Bode, Wohin geht die kirchliche Jugendarbeit? in: „Lebendiges Zeugnis“ 65(2010), S. 200-204.

9 Vgl. N. Possmann, Jugendpastoral..., S. 154-155; H. Haslinger, Selbstverwirklichung als Glaubensziel, in: „Jugend \& Kirche. Fachzeitschrift für Jugendarbeit und Jugendpastoral“ 28(1994/95), H. 2, S. 2-9.

10 Vgl. dazu H. Haslinger, „Um des Menschen willen!“ Worum es in der Jugendarbeit geht, wenn Gott ins Spiel kommt, in: Diakonische Jugendarbeit. Option für die Jugend und Option von Jugendlichen, Hrsg. G. Kruip, H. Hobelsberger, A. Gralla, München 1999, S. 57-82. 
Von der Seite der Jugendlichen werden an die Glaubensgemeinschaften (Kirchen) folgenden Behauptungen vorgeworfen: Sie freuen sich keinen „Vertrauensvorschuss“ mehr wie zuvor und werden nach ihren „Brauchbarkeit“ für die Existenz bewertet. Die Auswertung der Kirche liegt, nach Ralf Sauer, vom laufenden Eindruck in der Öffentlichkeit. Dort gilt sie aber vielmehr als unzeitgemäß, nicht immer zuverlässig, nicht modern, innerlich entzweit, an der gegenwärtigen gesellschaftlich-politischen Szene kaum anwesend ${ }^{11}$.

Einige junge Leute beschweren sich, dass die Seelsorger sie nicht so wollen, wie sie sind, aber wie sie nach einem "veralteten Stereotyp“ den „Bischöfen“ und den „Alten“ sein sollten. Nach ihnen macht die persönliche Existenz den zu schwachen Zusammenhang an die Pfarrgemeinde nicht reicher, sondern durch Anordnungen, Zuweisungen, Einwände und Ermahnungen vielmehr enger. Eine große Gruppe von jungen Menschen glaubt, dass die gegenwärtigen Andachten sehr ermüdend sind und ihnen nichts geben ${ }^{12}$. Aus ihrer Sicht setzen sie sich auch mit ihren Fragen hinsichtlich Öffentlichkeit, Politik, Milieu, Frieden nicht durch. Sie glauben, dass eine solche „Aktivität“- in den Augen der Kirchenhierarchie - weitab vom tatsächlich „Religiösen“ liegt (Vgl. Apg 4,32) ${ }^{13}$. Die Jugend betont auch, dass sie über die Sexualität ganz anders (oder zumindest offener) als die Kirche redet und denkt. Sie ist noch weiter leibfeindlich, sieht Sexualität gleich in Verbindung mit Sünde, prüft auch die Intimsphäre ${ }^{14}$. In der Tat werden wiederum schwere Sünden, wie öffentliches Unrecht, Ausbeutung

11 Vgl. R. Sauer, Die Kunst, Gott zu feiern..., S. 25-27; H. J. Höhn, Fremde Heimat Kirche..., S. 118-119; N. Possmann, Jugendpastoral..., S. 155-156.

12 Vgl. P. M. Zulehner, Mitgift. Autobiografisches anderer Art, Ostfildern 2014, S. 267; S. Knobloch, Gottesleere?..., S. 128-130.

13 Vgl. Franziskus, Evangelii gaudium, Rom 2014, Nr 31.

14 Vgl. P. M. Zulehner, Mitgift..., S. 279-283; B. Roebben, Moralpädagogische Überlegungen im Hinblick auf die Entwicklung christlicher Identität im Kulturwandel, in: Christwerden im Kulturwandel. Analysen, Themen und Optionen für Religionspädagogik und Praktische Theologie. Ein Handbuch, Hrsg. Th. Schreijäck, Freiburg-Basel-Wien 2001, S. 248-250. 
der Schöpfung, Formen nationaler Streiten, Ausländerhass durch sie vertragt und geschmälert ${ }^{15}$.

Es ist zu beachten, dass solche Meinungen nicht von Befangenheiten ungebunden sind. Doch nach den jungen Leuten, nähren sie viele Ursachen, auch offizielle Entscheidungen der Kirche und falsche Mediendarstellungen, und machen sie begreiflich ${ }^{16}$.

\section{Die Bedürfnisse der heutigen Jugend}

Die Behauptungen der jungen Menschen zeigen, dass sie die Kirche heute als Glaubensgemeinschaft nicht mehr - durch von oben auferlegte Gebote - an sich binden kann, darf sie aber auch nicht mit einfachen Werbevorgehensweisen anlocken. Ist die Jugend gegenwärtig wahrhaftig „privilegierte Aufgabe der Kirche“, dann muss sich diese erkundigen, was Jugend bedürft. Sie verkraftet, laut Patrick Höring, ihre Lebensaufgaben pragmatisch, nimmt aber doch wahr, dass ihr an vielem mangelt. Häufig fehlt ihr noch viel mehr, als sie wirklich will ${ }^{17}$.

Manche Heranwachsenden bemerken zuerst in ihrer eigenen Selbstverwirklichung lediglich eine Gestalt von wachsender Selbstsüchtigkeit. Auf der anderen Seite ist es grundlegende Schwierigkeit der Persönlichkeitsbildung, derzeit eine „Ich-Schwäche ${ }^{\text {“18. }}$. Eine große Gruppe von Jugendlichen hat nie zu sich selbst gefunden, weil ihr in der Kindheit das unentbehrliche Vertrauen zu Hause mangelte. Gesellschaftliche Unterstützung und die Werteharmonie einer früher hermetischen Glaubensgemeinschaft sind gefallen. Sie müssen die

\footnotetext{
15 Vgl. Ch. Friesl, Th. Hofer, R. Wieser, Die Österreicher/-innen und die Politik, in: Die Österreicher innen. Wertewandel 1990-2008, Hrsg. Ch. Friesl, R. Polak, U. Hamachers-Zuba, Wien 2009, S. 281-282.

16 Vgl. P. M. Zulehner, Kirche umbauen..., S. 91-92; F. H. Tenbruck, Die Kirchengemeinde in der entkirchlichten Gesellschaft, in: Soziologie der Kirchengemeinde, Hrsg. D. Goldschmidt, F. Greiner, H. Schelscky, Stuttgart 1960, S. 122-132.

17 Vgl. P. C. Höring, Kirchliche Jugendarbeit vor dem Aus?: einige Überlegungen angesichts aktueller Herausforderungen, in: „Stimmen der Zeit“231(2013), S. 85-90; S. Wahle, Grundlegung einer Theologie der Sakramentenfeiern, in: Heil erfahren..., S. 9.

18 Vgl. A. Wollbold, Handbuch der Gemeinde..., S. 290.
} 
eigene Position, ihre Identität, aufsuchen, oder sie werden von einer ganzen Menge und den Kommunikationsmitteln willenlos erobern. Anzeichen mangelnder Identität sind: persönliche Befürchtung, fehlender Einsatzmut, Unduldsamkeit, Empfindlichkeit für einflussreiche Personen und Pakte, Herden- und Anpassungsmentalität. Das voreilige Fliehen aus dem Familienleben, manche Methode von Widerstand, Provokation oder Faulheit, sind wie Hilferufe nach mehr eigener Bedeutung ${ }^{19}$.

Weil aktuell in vielen Elternhäusern häufig keine guten Verbindungen herrschen ${ }^{20}$, entkommen junge Leute sehr oft etwas zu früh in Zweierkontakte, um endlich mehr Akzeptanz zu erfahren. Für viele Teenager sind gegenwärtig unbeschränkte Weisen des Zusammenseins annehmbar. Die Intimbeziehung hat generell eine minderwertigere Bedeutung, als alte Leute befürchten. Bestimmt wird sie in der Tat nach sorgfältiger Partnerwahl nicht als bedeutendste Gestalt der Verbindung angesehen, aber vielmehr als eine der normalen Arten der Annäherung. Mit Übeltat (oder Sünde), so nehmen es viele junge Leute wahr, hat das nichts zu tun ${ }^{21}$.

Im Gegensatz zu vielen Meinungen, die junge Menschen als totale Individualisten schildern, kann man, laut Hans Hobelsberger, unter vielen von ihnen eine große Suche nach Gemeinschaft feststellen. Sie wollen aber keine Teams, die dirigiert werden. Im Gegenteil Sie wollen eine "Truppe“, in der sie souverän unter sich sind. Sie erstreben „Sicherheitsorte“ zum Entkommen vor den permanenten Ansprüchen der „gewaltigen“ Welt, die etwas Gefährliches an sich hat. Selbstverständlich verhüllt man ihnen, dass man ausgerechnet

\footnotetext{
19 Vgl. Ch. Friesl, Tausend Leben könnt' ich leben. Werthaltungen und Lebensstile österreichischer Jugendlicher in der modernen Gesellschaft, in: „Heiliger Dienst“ 49(1995), S. 13ff.

20 Vgl. A. Wollbold, Handbuch der Gemeinde..., S. 287-289.

21 Vgl. M. M. Lintner, Den Eros entgiften. Plädoyer für eine Tragfähige Sexualmoral und Beziehungsethik, Innsbruck-Wien 2011, S. 15-18; H. Kohler-Spiegel, Christ- und Christwerden im Kulturwandel, in: Christwerden im Kulturwandel..., S. 331, 344-350.
} 
von einer Gruppe nicht nur etwas fordern darf, aber dass man auch etwas opfern muss ${ }^{22}$.

Es ist auch möglich darauf hinzuweisen, dass Jugendliche gegenwärtig weniger risikobereit sind. Sie sind ebenfalls im Hinblick auf ihren Lebenslauf und ihre Lebenspläne mehr als jemals auf sich selbst gestellt, d. h. sie müssen derzeit die Elemente der Existenz in dieser Weise auf eigene Faust entscheiden und organisieren. Hierbei geraten sie in ein Dilemma: auf der einen Seite wird ihnen zugestanden, ihre Existenz nach eigenen Lebensregeln und Ideen in Gang zu setzen, im Gegensatz dazu platzen sie an sozialen Gesamtvoraussetzungen, denen gegenüber sie sich besinnungslos empfinden. Es kommt zu Politikverdruss sowie zu Glaubensgemeinschaftsverdruss ${ }^{23}$.

Viele Untersuchungen weisen darauf hin, dass heute junge Menschen ebenso sehr sachlich sind. Sie scheinen am besten zu wissen, was gut fürs Leben ist. Sie streben auch nach festen und gelungenen Liebesverhältnissen. Für sie sind, nach Christian Friesl, Freundschaft, aber auch Heirat und Familie (trotz alternativer Richtungen in der Öffentlichkeit), die Privatheit, für die sie viel einzusetzen bereit sind, die bevorstehende Laufbahn und ein einträchtiges Zusammenleben etc., von großer Bedeutung ${ }^{24}$. Auf der anderen Seite lernen sie aber ein Lebenskreis, in dem Verbindungen stets in Brüche gehen, Berufe verändert werden (müssen), die Erde gewaltsam vernichtet wird, kennen. Vieles, was in der Gesellschaft geschieht und ihre Zukunft gravierend gefährdet, zeigt sich ihnen absurd ${ }^{25}$.

Die durgeführten Forschungen zeigen, laut Stefan Knobloch, auch, das viele Jugendliche allerdings an einen Gott glauben, aber nicht,

${ }^{22}$ Vgl. H. Hobelsberger, Jugendgruppe, in: Lexikon..., S. 793-794; H. Steinkampf (Hrsg.), Kirchliche Jugendarbeit in Grundbegriffen, Düsseldorf-München 1985, S. 99-110.

${ }^{23}$ Vgl. Ch. Friesl, Tausend Leben könnt' ich leben..., S. 11; Ch. Friesl, Th. Hofer, R. Wieser, Die Osterreicher/-innen..., S. 281.

${ }^{24}$ Vgl. Ch. Friesl, Tausend Leben könnt' ich leben..., S. 10; D. Baacke, W. Ferchhoff, Jugendkulturen, in: Lexikon der Pastoral..., S. 794-795.

${ }^{25}$ Vgl. D. Baacke, W. Ferchhoff, Jugendarbeit, Jugendbildung, in: Lexikon..., S. 787-788. 
wie er seitens der verschiedenen Glaubensgemeinschaften angekündigt wird. Außerdem hat für sie die Person Christi eine Bedeutung, aber nur als außergewöhnlicher Mensch, ähnlich wie Gandhi oder Mahomet, nicht aber als Sohn Gottes. Religiöses Anmachen wird von vielen Teenagern verworfen, weil Pfarrgemeinde häufig nicht mehr als etwas Besonderes und Zuverlässiges erlebt wird ${ }^{26}$. Im anderen Sinn betonen junge Menschen, dass das private Verlangen nach Gott im engsten Kreise der religiösen Glaubensgemeinschaften kaum gestillt wird. Sie erleben die bergende Reichweite Gottes weder in der Verkündigung noch im Gottesdienst. Sie erfahren vielmehr ein begriffliches, geistliches Gottesbild, einen Gott, der hauptsächlich verlangt und beurteilt, eine Gottesanbetung, die zu einer Zeremonie geworden ist und mit der Existenz fast nichts zu tun hat. Ihre Sympathie für Christus findet in der Pfarrgemeinde keine entsprechende Analogie. Es mangelt ihnen an Muster einer vollkommenen Jesusnachfolge. Die Größen unserer Zeit (für die Christen: die Heiligen) entdecken sie vielmehr woanders, nicht in der Pfarrgemeinde ${ }^{27}$.

Aus den Äußerungen vieler Heranwachsenden zeigt sich auch, dass bei ihnen ein großer Instinkt zu Unabhängigkeit herrscht. Sie empfinden, unendlich „bevormundet“ zu werden. Auf der anderen Seite bemerken sie nicht, wie oft sie sich neuen falschen Passionen ergeben. Beispielsweise sind sie für Kommerz mühelos zu bewerbende Klienten. Man beobachtet nur die Inklination für die Markenjeans und T-Shirts, endlos neue Innovationen im Handel von Sportgeräten und Spielen usw. Sie verwerfen unmoderne Regeln, unterjochen sich aber Benehmensmustern, wie sie ihnen von ihren Illustrierten und „Helden“ fast „unvermeidbar“ dargestellt werden. Zugleich sehnen sich die Jugendlichen nach einer Existenz in Harmonie, mit mehr

\footnotetext{
26 Vgl. S. Knobloch, Gottesleere?..., S. 85-87; J. Sobrino, Überheblichkeit und Demut. Anmerkung zum gegenwärtigen Zustand der Kirche, in: „Concilium“ 46 (2010), H. 4, S. $478-484$.

27 Vgl. R. Sauer, Die Kunst, Gott zu feiern..., S. 177-179; H. J. Höhn, Fremde Heimat Kirche..., S. 128-129; S. Leimgruber, Jugend und Liturgie, in: „Keryks. Internationale religionspädagogisch-katechetische Rundschau“1(2002), S. 141-154.
} 
Solidarität und mehr Herzlichkeit für die Natur. Eine Welt, besonders auch eine Berufswelt, in der es lediglich handeln muss. Viele sehnen sich auch nach einer „zeitgemäßen“ kirchlichen Glaubensgemeinschaft, die solches anerkennt, bevorzugt und mit aller ihrer Mühe in der Welt zu erfüllen erstrebt ${ }^{28}$.

\section{Die wichtigsten Aufgaben der Jugendpastoral in den Pfarrgemeinden}

Die obigen Aussagen der jungen Leute zeigen, dass es gegenwärtig vor jeder Jugendseelsorge in den einzelnen Pfarrgemeinden sehr große Herausforderungen stehen ${ }^{29}$. In ihnen geht es aber nicht bloß um die Glaubensgemeinschaft, gewissermaßen in der Art einer Werbekampagne „anziehend“ für Teenager zu machen, aber nachzudenken, welche Bedeutung Kirche der Jugend gegenüber hat und welchen unersetzlichen Raum Jugend in der Errichtung einer Gemeinde, im Leben einer Pfarre, haben muss, damit hier das Apostolat Jesu (Vgl. AA 12) verwirklicht werden $\mathrm{kann}^{30}$.

Viele Seelsorger betonen, dass die Heranwachsenden gegenwärtig in jeder Kirchengemeinde besonders ernst genommen werden müssen. In besonderer Weise wollen sie ernst genommen werden mit ihren Fragen nach Partnerschaft, Milieu, Friede, Freiheit, eigene Weisen der Gottesverbindung usw. Allerdings muss auch, nach Leo Karrer, ihr Engagement ernst genommen werden ${ }^{31}$. Es genügt nicht nur ein Vertreter im Pfarrgemeinderat, über den man „Zuverlässig“

\footnotetext{
28 Vgl. B. Kranemann, Die Feier..., S. 97-98; W. Tzscheetzsch, Jugend. Praktisch-theologisch, in: Lexikon der Pastoral..., S. 786-787.

${ }^{29}$ Vgl. B. Piwowarczyk, Die heutige Jugend fordert uns heraus, in: „Klerusblatt“ 85(2005), S. 173-176; Ch. Friesl, Die postchristliche Jugend. Herausforderung für Kirche, Gemeinde und Liturgie, in: „Heiliger Dienst“ 49(1995), S. 19-24.

30 Vgl. W. Tzscheetzsch, Jugend. Praktisch...., S. 786; P. M. Zulehner, W. Müller, B. Sieberer (Hrsg.), Der Reichtum der Kirche sind ihre Menschen..., S. 78-79; A. Saberschinsky, Der gefeierte Glaube. Einführung in die Liturgiewissenschaft, Freiburg-Basel-Wien 2006, S. 162-163; L. Karer, Handbuch..., S. 265-266.

31 Vgl. L. Karer, Handbuch..., S. 301-302; W. Tzscheetzsch, Jugend. Praktisch...., S. 786.
} 
Anweisungen oder Klagen an die Jugend übermitteln kann. Heranwachsende müssen tatsächlich „Stimme“ haben. Nicht nur, was die direkte Jugendarbeit betrifft, sondern auch das Gemeindebestehen. Sie sollten als „Dopingmittel“ und „Regulativ“ gehört werden ${ }^{32}$.

Es lohnt sich ebenfalls, laut Leo Karer, den Jugendlichen in der Pfarrgemeinden Plätze zur Aktivität zu errichten ${ }^{33}$. Am Anfang ist die Rede von den Plätzen für jugendgerechtes Beisammensein mit der Gelegenheit eigener Entwicklung und Leitung. Ein „Heim“ im wahrsten Sinn des Wortes. Noch bedeutender sind aber Plätze zur Entwicklung der Individualität, Selbstfindung, Verkehrspotenz, für gesellschaftliche Einstellungen sowie für spätere Beziehungen. Entwicklung kommt nur durch Bemühung zustande. Das bedeutet selbstverständlich auch eine Gefahr, die man absichtlich annehmen muss. Ähnlich ist das Erfahren der persönlichen Beschränkungen erzieherisch. Derartige Wirkungsfelder zeigen sich nicht nur in schwungvoll zugelassenen Handlungsmethoden, aber noch mehr in der Atmosphäre des Zutrauens, der Redlichkeit und im Acht vor den Heranwachsenden ${ }^{34}$.

Aus Furcht, junge Leute zu verwirken, werden sie aktuell vielmehr unter- als überfordert. Man vertraut auf die Heranwachsende kaum noch, dass sie sich gleichermaßen gegen die „-ismen“, wie Konsumismus, Hedonismus, Säkularismus, Individualismus, beschweren. Wo Teenager aufgeregt werden, enthüllt man, wenn sie statt aller „Milieuschädigung“ doch noch etwas Gutes behalten haben ${ }^{35}$.

Die Anforderungen sollten hauptsächlich folgendermaßen gelten:

\footnotetext{
32 Vgl. H. J. Höhn, Fremde Heimat Kirche..., S. 129; H. Hobelsberger, Jugendgruppe..., S. $793-794$.

33 Vgl. L. Karer, Handbuch..., S. 165-166 und 168; H. Hobelsberger, Exodus und Landnahme: neue Sozialformen in der Jugendpastoral, in: „Herder-Korrespondenz/ Spezial“" (2011), S. 40-41.

34 Vgl. Ch. Friesl, Kirche auf der Suche nach der Jugend. Zum zukünftigen Verhältnis von Jugend, Glaube und Kiche, in: „Jugend und Kirche“ 23(1989/90), H. 3, S. 2-6; H. Hobelsberger, Jugendpastoral..., S. 799-780.

35 Vgl. R. Sauer, Die Kunst, Gott zu feiern..., S. 26-27; A. Saberschinsky, Der gefeierte Glaube..., S. 173-174.
} 
- Anforderung zur Kritik: Man unterstellt den jungen Leuten häufig eine unbegrenzte Jagd nach Beurteilungen. Sie haben dabei ein neues enormes Potenzial, Positionen und Anlässe anderer zu entdecken. Ihre Einsprüche gegen altmodische Überlieferung zwingt die Kirchengemeinde zur Revision des allzu Gewohnten. Man kann sagen, dass eine faire Kritik dieser Form sich anzueignen selbst auch unterstützt wird, Falschdemokratie und geheuchelte Rechte auf eigene Meinung in Pfarrgemeinde und in der Öffentlichkeit zu demaskieren und zu einer authentischen Mitverantwortung zu heranzubilden ${ }^{36}$.

- Anforderung zu mehr Unabhängigkeit: Das kommt hauptsächlich durch Übertragen eigener Verantwortung zustande. Der Mensch entwickelt sich ununterbrochen dann am besten, wenn er für etwas verantwortlich ist. Dies ist möglich in Entwürfen, die bestimmt nicht verordnet werden dürfen, aber aus dem Interessensgebiet der Heranwachsenden bereitwillig selber kommen sollen. Spätere Jugendtätigkeit wird absolut stark projektorientiert sein. Das birgt die Gefahr der Kurzfristigkeit in sich, spricht aber erneut immer neue Schichten der jungen Leute $\mathrm{an}^{37}$.

Eine weitere wichtige Forderung ist mit dem Grund, für die jungen Leute in der Pfarrgemeinde verschiedene religiöse Angebote einzurichten, gebunden. Es ist zu beachten, dass die Teenager oft dem Gemeindeleben fern bleiben, weil ihnen zu wenig "Geistigkeit“ angeboten wird. Die Reflexion über Gott (eingefügt in die Reflexionen über den Sinn des Lebens) ist wichtiger, als wir ahnen. Jugendanbetungen, karitativen Aktionen, Fußwallfahrten mit klaren Inhalten locken überraschend komplett neue Gruppen Teenager an. Die Möglichkeiten für jugendgerecht eingerichtete Andachten sollten

\footnotetext{
36 Vgl. N. Köbel, Jugend - Identität - Kirche. Eine erziehungswissenschaftliche Rekonstruktion kirchlicher Orientierungen im Jugendalter, Frankfurt a. M. 2009, S. 41-42.

37 Vgl. B. Grom, „Vom Egotrip zur Solidarität“, in: „Stimmen der Zeit“ 118(1993), S. 146.
} 
gegeben sein, aber ebenso mitgewirkt werden ${ }^{38}$. In geistlichen Vorschlägen sollten die unterschiedlichen Anbetungs-, Nachdenkensund Andachtsanlässe nicht ausgedehnt, sondern es sollte jede auf den Lebenszusammenhang der Heranwachsenden und ihre Lebensweise angepasst werden, sich entsprechend auszudrucken und zu ehren ${ }^{39}$.

Einige Jugendseelsorger glauben, dass manchen jungen Menschen soll gegenwärtig der Status des „Katechumenats“ zugestehen ${ }^{40}$. Verbindet ist es mit der Lage der Katechumenen in der alten Kirche. Eine große Gruppe von Jugendlichen befindet sich, ebenfalls wie diese vormals, am Beginn der Evangelisierung. An Katechumenen kann und muss man noch nicht völlige Erfordernisse legen, die sich zu den Aufgaben eines erwachsenen Gläubiger beziehen. Für sie hat, nach Ralf Sauer, die Glaubensgemeinde ebenfalls ständig schon verschiedene Gestaltungen des Gottesdienstes gekannt, um sie Schritt für Schritt in das ganze Mysterium anzuleiten ${ }^{41}$.

Auf der anderen Seite muss aber das Katechumenat ein Ort haben, ein Ziel, auf das je nach Bedingungen und merkbarem Vorwärtskommen zugegangen wird. Katechumenen benötigen Betreuer (Paten), jedoch solche, die zur persönlichen Handlung beanspruchen, die Vorbild sind und doch auch gemeinsam Effekte suchen. Nach Paul Zulehner, aus den noch bestehenden Resten des Volkskirchentums bieten sich für dieses Ziel besonders drei Ereignisse an: Vorbereitung zu der ersten heiligen Kommunion, Firmkatechumenat und

38 Vgl. R. Sauer, Die Kunst, Gott zu feiern..., S. 233-235; L. Karer, Handbuch..., S. 250; J. Knupp, Jugendwallfahrten, in: Lexikon der Pastoral..., S. 802.

39 Vgl. R. Sauer, Die Kunst, Gott zu feiern..., S. 256-261; J. Bärsch, Jugendgottesdienst, in: Lexikon der Pastoral..., S. 791-792; J. Knupp, Jugendexerzitien, in: Lexikon der Pastoral..., S. 791.

40 Vgl. R. Sauer, Die Kunst, Gott zu feiern..., S. 176-178 und 206-209; M. Ball, Katechumenat. Pastoral, in: Lexikon der Pastoral..., S. 821-822.

41 Vgl. R. Sauer, Die Kunst, Gott zu feiern..., S. 270-276; M. Klöckener, Die entfremdete Beziehung zwischen Jugendlichen und Liturgie, in: „Liturgiches Jahrbuch“ 39(1989), S. 228-252. 
Ehevorbereitung ${ }^{42}$. Diese stufenweise Handlung fordert auch, dass es in jeder Gemeinde eine „fortgeschrittene“, verantwortliche Kerngemeinschaft gibt. Sie darf sich nicht besser empfinden als die anderen. Aber ihr „reizendes“ Beispiel wird zur Reflexion ermuntern. Die Haftung, die sie für andere erträgt, wird sie selbst „unterwegs halten“ und neu anspornen. Zusammenarbeit von Gruppen ereignet sich stets durch Annäherung und so ebenfalls in Gruppen ${ }^{43}$.

Man soll sich auch um eine Kontaktperson für die jungen Menschen in der Gemeinde kümmern, weil dort Jugendseelsorge oft mit einer Bezugsperson steht und fällt. Das ist, nach Dieter Baacke und Wilfried Ferchhoff, eine sehr wichtige Aufgabe für jeden Jugendseelsorger und auch für Jugendleiter. Die Erfordernisse an diese sind mannigfaltig und sehr groß. Sie benötigen nicht nur eine ekklesial gültige Zuständigkeitskompetenz, aber auch eine individuell gelernte Fähigkeitskompeten $z^{44}$. Zuallererst müssen sie in umsichtiger Art Teenager begleiten und sich durchaus auf ihrer Seite befinden. Danach müssen sie auch Verteidiger der Jugendlichen der Gemeinde gegenüber sein. Sie werden für die Anwendung des Freiraumes, offensichtlich im Ernstfall auch für Pannen, verantwortlich gemacht. Die Sache liegt in der Entscheidung, in der Schulung. Solche Kontaktpersonen gehören "gesichert“, man muss ihnen Vertrauen spenden und ihnen Geleit geben ${ }^{45}$.

Es ist auch wichtig, nach Hans Hobelberger, eine "glaubwürdige“ Glaubensgemeinschaft den Heranwachsenden hinzudeuten. Die jungen Menschen sind heutzutage sehr feinfühlig, was die Darstellung

\footnotetext{
${ }^{42}$ Vgl. P. M. Zulehner, "Seht her, nun mache ich etwas Neues“. Wohin sich die Kirchen wandeln müssen, Ostfildern 2011, S. 130-142.

43 Vgl. dazu J. Bärsch, Jugendliche in der Liturgie, in: „Heiliger Dienst“ 49(1995), S. 1-57; Ders., Jugendgottesdienst...,S. 92.

44 Vgl. D. Baacke, W. Ferchhoff, Jugendarbeit..., S. 788; H. Stenger (Hrgs.), Eignung für die Berufe der Kirche, Freiburg-Basel-Wien 1990, S. 34ff. und 47ff.; P. Scheuchenpflug, Junge Menschen begleiten und fördern, in: Gemeinde gestalten, Hrsg. K. Baumgartner, Regensburg 1999, S. 141-172.

45 Vgl. H. Stenger (Hrgs.), Eignung für die Berufe..., S. 37; L. Karer, Handbuch..., S. $258-259 ; 286$.
} 
der Kirche in ihrer Pfarre betrifft, die sie jeden Tag und jeden Sonntag erhalten. Jugendliche sind ganz speziell für ein Auseinanderklaffen zwischen religiöser Darlegung einerseits und der realen Existenz einer Pfarrgemeinde andererseits empfindlich. Es benötigt von hier eine glaubwürdige Glaubensgemeinschaft, die sich erstrebt, vor allem nach den Grundgesetzen der Bergpredigt zu existieren, um Heranwachsende für die Pfarre, für Jesus Christus und für das Reich Gottes betören zu können (Vgl. Phil 2,6-11). Junge Leute müssten in der wirklichen Kirchengemeinde ein bisschen von einer "redemptiven“, erlösenden Umgebung, erfahren (Vgl. GS 44) ${ }^{46}$.

Gegenwärtig müssen wir auch, laut Anette Schavan, darauf achten, den Heranwachsende solche Kirche Darstellung zu offenbaren, zu die sie aufs Neue zukommen möchten ${ }^{47}$. Die Allgemeinstimmung in der katholischen Glaubensgemeinschaft ist aktuell generell bedrückend. Die „Lebensumstände“ in der Kirche können wir nicht sofort verändern, dennoch können wir unsere Pfarrgemeinden vorwiegend persönlich entwickeln. Ausgerechnet um der Teenager willen ginge es, laut Leo Karer, darum, Gemeinden zu einer Stelle freundschaftlicher Kommune zu erschaffen ${ }^{48}$. Das sollten Orte der Begegnung sein, wo sich alle Menschen, auch derartige, die andersartig betrachten, anders denken oder am Rande der Gesellschaft und in Krisen sind, wie zu Hause fühlen. Eine Pfarrgemeinschaft, die mehr das Gesetz Christi als eine kasuistische Ethik zur Regel ihrer Verhaltensweise macht. Gemeinden können mehr, als sie sich zutrauen ${ }^{49}$.

\footnotetext{
46 Vgl. H. Hobelsberger, Jugendpastoral..., S. 799-800; S. Knobloch, Gottesleere?..., S. 107; P. M. Zulehner, Mitgift..., S. 265; H. J. Höhn, Fremde Heimat Kirche..., S. 131-132.

47 Vgl. A. Schavan, In Zukunft glauben: Christentum in der jungen Generation, in: Kreuzungen. Christliche Existenz im Diskurs, Hrsg. H. Baldur, G. Berghaus, Mülheim/Ruhr 2002, S. 385-389; A. Foitzik, Hoffen auf die Jugend? in: „Herder-Korrespondenz" 66(2012), S. 55-57.

48 Vgl. L. Karer, Handbuch..., S. 271-272 und 313-314; H. Hobelsberger, Jugendpastoral..., S. 141-172.

49 Vgl. H. J. Höhn, Fremde Heimat Kirche..., S. 136-138; N. Mette, Gemeinde. Praktisch-theologisch, in: Lexikon der Pastoral..., S. 573-574.
} 
Dennoch darf sich eine so erfahrbare Glaubensgemeinschaft am Ort nicht als ein „einmaliger" seelsorglicher Ort empfinden, der besser ist als andere. Selbst die Ortskirche sollte immer eine Kirche in Verbindung mit der ganzen Kirche sein. Wir sollten uns das Anschauen auf die katholische Kirche nicht durch schicksalhafte Begebenheiten oder durch tendenzielle Medienmitteilungen vermiesen lassen. Auch die jungen Menschen sollten, nach Werner Tzscheetzsch, die Augen für die prophetische Funktion aufmachen, die die katholische Kirche auch gegenwärtig für die Welt leistet. Betrachten wir lediglich den kirchlichen Einsatz für den Frieden oder die nachdrückliche Option für die Armen ausgerechnet in der Dritten Welt. Man denke an die ansteigende Bedeutung der Kirch(en) in Europa im Hinblick auf Völkerwanderung, Einwanderer oder Flüchtlinge, etc ${ }^{50}$.

\section{Zusammenfassung}

Zusammenfassend sollte betont werden, dass manchmal das gegenwärtige weltweite Bild der katholischen Kirche und ihre Richtungsstreitigkeiten auch auf die kritische Beurteilung der Heranwachsenden herausfordern. In dieser pastoraltheologischen Reflexion wollten wir zeigen, dass die Kirche auch diese Kritik positiv nutzen könnte. Auch sie (vor allem wir - Mitglieder dieser Glaubensgemeinschaft) braucht immer Selbstkritik, vor allem zur Frage, was müsste sich noch verbessern, dass ihr menschliches Kleid wieder mehr vom göttlichen Mysterium durchstrahlen lässt? Und dafür sollten wir auch der jungen Menschen anmutig sein, dass sie uns häufig rücksichtlos auf Veränderungsbedürftiges in unserer Glaubensgemeinschaft hinweisen.

\section{Streszczenie:}

Przemiany dokonujące się dziś na świecie, ich zakres i złożoność oraz skutki, kształtują postawy społeczne jednostek ludzkich, grup społecznych

\footnotetext{
50 Vgl. W. Tzscheetzsch, Jugendorganisationen, in: Lexikon der Pastoral, Hrsg. K. Baumgartner, P. Scheuchenpflug, Bd. 1 A-Ki, Freiburg-Basel-Wien 2002, S. 796 798; J. B. Metz, Gottespassion, Freiburg-Basel-Wien 1991, S. 48ff.; J. L. Allen, Das neue Gesicht der Kirche. Die Zukunft des Katholizismus, Gütersloh 2010, S. 307-308.
} 
i całych społeczeństw. Mają one również olbrzymi wpływ na funkcjonowanie Kościoła i ludzi wierzących, w tym także na ludzi młodych. Aktualnie, w rozwijających się społeczeństwach, zainteresowanie się problematyką i warunkami funkcjonowania w nich młodego pokolenia, zależy od określonej kultury społecznej i ekonomicznej. Należy jednak podkreślić, że zagadnienie to stanowiło zawsze bardzo istotny element posługi duszpasterskiej Kościoła. Kościół starał się bowiem stale wypełniać to zadanie wszystkimi dostępnymi mu sposobami. Warto również zaznaczyć, że korzystający dziś z posługi duszpasterskiej Kościoła młodzi ludzie odznaczają się bardzo zróżnicowanym poziomem zainteresowania Ewangelią, a w konsekwencji i różnym poziomem życia religijnego. Można to zauważyć wśród młodzieży korzystającej ze szkolnych lekcji religii (np. obserwując ich postawy wobec własnej wspólnoty parafialnej), jak również wsłuchując się w ich wypowiedzi na temat Kościoła i religii w środkach społecznego przekazu.

Niniejszy artykuł, oparty na analizie literatury teologicznopastoralnej (ale także socjologicznej) wybranych autorów z krajów niemieckojęcznych (Niemcy, Austria, Szwajcaria), pragnie zwrócić uwagę na to, jak oni widzą dzisiaj problemy wzajemnych relacji młodych ludzi i Kościoła w ich krajach. Składa się on z trzech rozdziałów. Pierwszy z nich próbuje odpowiedzieć na pytanie, dlaczego wielu młodych ludzi nie postrzega dziś wspólnoty Kościoła jako swojego „domu“. Drugi z kolei przedstawia najważniejsze potrzeby współczesnej młodzieży, opierając się na wypowiedziach jej przedstawicieli, zanotowanych przez cytowanych autorów, a trzeci podaje najważniejsze zadania, jakie stoją dziś przed duszpasterstwem młodzieży w parafiach. Należy również podkreślić, że przyczynkowy charakter tego opracowania powoduje, że nie pretenduje ono do szerszego i kompleksowego spojrzenia na powyższy problem.

\section{Summary}

This article, based on an analysis of pastoral literature (but also sociological and) selected authors from Germany, Austria, Switzerland, wishes to draw attention to how they see today the problems of young people and the relationship of the Church in their countries. It consists of three chapters. The first one trying to answer the question of why many young people do not perceive the Church as a community today of his "home”. The second, in turn, presents the most important needs of today's youth, based on the statements of its agents, recorded by cited authors, and the third gives the most important task, which stand today before pastoral care of the young 
in parishes. It should also be pointed out that this study does not pretend it to a broader and comprehensive look at this issue.

Słowa klucze: Kościół; młodzież; parafia; duszpasterstwo młodzieży; katechumenat.

Key words: The Church; young people; the parish; pastoral care of the young, catechumenate

\section{Biogram:}

Dr hab. prof. UŚ, kapłan archidiecezji katowickiej. Pracuje w Wydziale Teologicznym UŚ w Katowicach w Katedrze Teologii Pastoralnej, Liturgiki, Homiletyki i Katechetyki. W latach 2011-2012 stypendysta Uniwersytetu Wiedeńskiego (Pastorales Forum). W 2012 roku otrzymał „Kardinal-BertramStipendium”, po wygraniu konkursu, ogłoszonego przez „Institut für ostdeutsche Kirchen- und Kulturgeschichte e.V” z siedzibą w Regensburgu. Jest autorem i redaktorem licznych książek. oraz ponad 100 artykułów naukowych z zakresu teologii pastoralnej i liturgiki.

\section{Bibliografia:}

Allen J. L., Das neue Gesicht der Kirche. Die Zukunft des Katholizismus, Gütersloh 2010.

Baacke D., Ferchhoff W., Jugendarbeit, Jugendbildung, in: Lexikon der Pastoral, Hrsg. K. Baumgartner, P. Scheuchenpflug, Bd. 1 A-Ki, Freiburg-Basel-Wien 2002, S. 787-788.

Ball M., Katechumenat. Pastoral, in: Lexikon der Pastoral, Hrsg. K. Baumgartner, P. Scheuchenpflug, Bd. 1 A-Ki, Freiburg-Basel-Wien 2002, S. 821-822.

Barz H., Religion ohne Institution? Eine Bilanz der sozialwissenschaftlichen Jugendforschung, Opladen 1992.

Baumgartner K. (Hrsg.), Gemeinde gestalten, Regensburg 1999.

Bärsch J., Jugendliche in der Liturgie, in: „Heiliger Dienst“ 49(1995), S. 1-57. Bärsch J., Jugendgottesdienst, in: Lexikon der Pastoral, Hrsg. K. Baumgartner, P. Scheuchenpflug, Bd. 1 A-Ki, Freiburg-Basel-Wien 2002, S. 791-792.

Bleistein R., Jugendpastoral - Entwurf einer Neukonzeption, in: „Katechetische Blätter“100(1975), S. 65-76. 
Baacke D., Ferchhoff W., Jugendkulturen, in: Lexikon der Pastoral, Hrsg. K. Baumgartner, P. Scheuchenpflug, Bd. 1 A-Ki, Freiburg-Basel-Wien 2002, S. 794-795.

Bleistein R., Jugend. Theologisch-pastoral, in: Lexikon der Pastoral, Hrsg. K. Baumgartner, P. Scheuchenpflug, Bd. 1 A-Ki, Freiburg-Basel-Wien 2002, S. 785-786.

Bode F. J., Wohin geht die kirchliche Jugendarbeit? in: „Lebendiges Zeugnis“ 65(2010), S. 200-207.

Ebertz M. N., Die sieben "Jugendtabus" der Kirche. Soziologische Aspekte eines problematisch gewordenen Verhältnisses, in: „Katechetische Blätter“ 124(1999), S. 406-410.

Foitzik A., Hoffen auf die Jugend? in: „Herder-Korrespondenz“ 66(2012), S. 55-57.

Friesl Ch., Kirche auf der Suche nach der Jugend. Zum zukünftigen Verhältnis von Jugend, Glaube und Kiche, in: ,Jugend und Kirche“23(1989/90), H. 3, S. 2-6.

Friesl Ch., Tausend Leben könnt' ich leben. Werthaltungen und Lebensstile österreichischer Jugendlicher in der modernen Gesellschaft, in: „Heiliger Dienst" 49(1995), S. 7-18.

Friesl Ch., Die postchristliche Jugend. Herausforderung für Kirche, Gemeinde und Liturgie, in: „Heiliger Dienst“ 49(1995), S. 19-27.

Friesl Ch., Hofer Th., Wieser R., Die Österreicher/-innen und die Politik, in: Die Österreicher innen. Wertewandel 1990-2008, Hrsg. Ch. Friesl, R. Polak, U. Hamachers-Zuba, Wien 2009, S. 207-293.

Grom B., „Vom Egotrip zur Solidarität“, in: „Stimmen der Zeit“ 118(1993), S. $145-146$.

Haslinger H., Selbstverwirklichung als Glaubensziel, in: „Jugend \& Kirche. Fachzeitschrift für Jugendarbeit und Jugendpastoral“ 28(1994/95), H. 2, S. 2-9.

Haslinger H., „Um des Menschen willen!“ Worum es in der Jugendarbeit geht, wenn Gott ins Spiel kommt, in: Diakonische Jugendarbeit. Option für die Jugend und Option von Jugendlichen, Hrsg. G. Kruip, H. Hobelsberger, A. Gralla, München 1999, S. 57-82.

Hobelsberger H., Exodus und Landnahme: neue Sozialformen in der Jugendpastoral, in: „Herder-Korrespondenz/Spezial“ (2011), S. 40-43.

Hobelsberger H., Jugendgruppe, in: Lexikon der Pastoral, Hrsg. K. Baumgartner, P. Scheuchenpflug, Bd. 1 A-Ki, Freiburg-Basel-Wien 2002, S. 793-794. 
Hobelsberger H., Jugendpastoral, in: Lexikon der Pastoral, Hrsg. K. Baumgartner, P. Scheuchenpflug, Bd. 1 A-Ki, Freiburg-Basel-Wien 2002, S. 799-780.

Höhn H. J., Fremde Heimat Kirche. Glauben in der Welt von heute, Freiburg-Basel-Wien 2012.

Höring P. C., Kirchliche Jugendarbeit vor dem Aus?: einige Überlegungen angesichts aktueller Herausforderungen, in: „Stimmen der Zeit“231(2013), S. 85-95.

Inglehart R., Kultureller Umbruch, Frankfurt-New York 1989.

Karer L., Handbuch der praktischen Gemeindearbeit, Freiburg-Basel-Wien 1990.

Klöckener M., Die entfremdete Beziehung zwischen Jugendlichen und Liturgie, „Liturgiches Jahrbuch“ 39(1989), S. 228-252.

Knobloch S., Gottesleere? Wider die Rede vom Verlust des Göttlichen, Ostfildern 2013.

Knupp J., Jugendexerzitien, in: Lexikon der Pastoral, Hrsg. K. Baumgartner, P. Scheuchenpflug, Bd. 1 A-Ki, Freiburg-Basel-Wien 2002, S. 791.

Knupp J., Jugendwallfahrten, in: Lexikon der Pastoral, Hrsg. K. Baumgartner, P. Scheuchenpflug, Bd. 1 A-Ki, Freiburg-Basel-Wien 2002, S. 802.

Kohler-Spiegel H., Christ- und Christwerden im Kulturwandel, in: Christwerden im Kulturwandel. Analysen, Themen und Optionen für Religionspädagogik und Praktische Theologie. Ein Handbuch, Hrsg. Th. Schreijäck, Freiburg-Basel-Wien 2001, S. 330-354.

Köbel N., Jugend - Identität - Kirche. Eine erziehungswissenschaftliche Rekonstruktion kirchlicher Orientierungen im Jugendalter, Frankfurt a. M. 2009.

Kranemann B., Die Feier der christlichen Initiation, in: Heil erfahren in den Sakramenten, H. Hoping, B. Kranemann, S. Wahle, N. Weidinger, Freiburg-Basel-Wien 2009, S. 52-103.

Leimgruber, S. Jugend und Liturgie, in: „Keryks. Internationale religionspädagogisch-katechetische Rundschau"1(2002), S. 141-154.

Lintner M. M., Den Eros entgiften. Plädoyer für eine Tragfähige Sexualmoral und Beziehungsethik, Innsbruck-Wien 2011.

Mette N., Gemeinde. Praktisch-theologisch, in: Lexikon der Pastoral, Hrsg. K. Baumgartner, P. Scheuchenpflug, Bd. 1 A-Ki, Freiburg-Basel-Wien 2002, S. 572-574.

Metz J. B., Gottespassion, Freiburg-Basel-Wien 1991.

Nauer D., Seelsorge. Sorge um die Seele, Stuttgart 2010. 
Piwowarczyk B., Die heutige Jugend fordert uns heraus, in: „Klerusblatt“ 85(2005), S. 173-177.

Possmann N., Jugendpastoral, in: Lexikon des Apostolats. Stichworte verantworteten Glaubens, Hrsg. F. Courth, Limburg 1995, S. 154-157.

Roebben B., Moralpädagogische Überlegungen im Hinblick auf die Entwicklung christlicher Identität im Kulturwandel, in: Christwerden im Kulturwandel. Analysen, Themen und Optionen für Religionspädagogik und Praktische Theologie. Ein Handbuch, Hrsg. Th. Schreijäck, Freiburg-Basel-Wien 2001, S. 248-274.

Saberschinsky A., Der gefeierte Glaube. Einführung in die Liturgiewissenschaft, Freiburg-Basel-Wien 2006.

Sauer R., Die Kunst, Gott zu feiern. Liturgie wiederentdecken und einüben, München 1996.

Schavan A., In Zukunft glauben: Christentum in der jungen Generation, in: Kreuzungen. Christliche Existenz im Diskurs, Hrsg. H. Baldur, G. Berghaus, Mülheim/Ruhr 2002, S. 385-399.

Scheuchenpflug P., Junge Menschen begleiten und fördern, in: Gemeinde gestalten, Hrsg. K. Baumgartner, Regensburg 1999, S. 141-172.

Schmidtchen G., Gottesdienst in einer rationalen Welt, Stuttgart-Freiburg 1973.

Sobrino J., Überheblichkeit und Demut. Anmerkung zum gegenwärtigen Zustand der Kirche, in: „Concilium“ 46 (2010), H. 4, S. 478-484.

Steinkampf H. (Hrsg.), Kirchliche Jugendarbeit in Grundbegriffen, Düsseldorf-München 1985, S. 99-110.

Stenger H. (Hrgs.), Eignung für die Berufe der Kirche, Freiburg-Basel-Wien 1990.

Tenbruck F. H., Die Kirchengemeinde in der entkirchlichten Gesellschaft, in: Soziologie der Kirchengemeinde, Hrsg. D. Goldschmidt, F. Greiner, H. Schelscky, Stuttgart 1960, S. 122-132.

Trapp E., Seelsorge - eine (andere) Pflegequalität?, in: „Pastoralblatt“ 10(2011), S. 300-306.

Tzscheetzsch W., Jugend. Praktisch-theologisch, in: Lexikon der Pastoral, Hrsg. K. Baumgartner, P. Scheuchenpflug, Bd. 1 A-Ki, Freiburg-Basel-Wien 2002, S. 786-787.

Tzscheetzsch W., Jugendorganisationen, in: Lexikon der Pastoral, Hrsg. K. Baumgartner, P. Scheuchenpflug, Bd. 1 A-Ki, Freiburg-Basel-Wien 2002, S. 796-798. 
Wahle S., Grundlegung einer Theologie der Sakramentenfeiern, in: Heil erfahren in den Sakramenten, H. Hoping, B. Kranemann, S. Wahle, N. Weidinger, Freiburg-Basel-Wien 2009, S. 5-51.

Wollbold A., Handbuch der Gemeinde-Pastoral, Regensburg 2004.

Wustmans H., Firmung oder die Frage nach der Autorität der Kirche im Erleben Jugendlicher, in: „Theologisch-praktische Quartalschrift" 158(2010), S. 371-380.

Zinnecker J., Jugend, Kirche und Religion. Aktuelle empirische Ergebniss und Entwicklungstendenzen, in: Religionsunterricht im Abseits? Das Spannungsfeld Jugend, Schule, Religion, Hrsg. G. Hilger, G. Reilly, München 1993, S. 114-119.

Zulehner P. M., Kirche umbauen - nicht totsparen, Kevelaer 2009.

Zulehner P. M., „Seht her, nun mache ich etwas Neues". Wohin sich die Kirchen wandeln müssen, Ostfildern 2011.

Zulehner P. M., Mitgift. Autobiografisches anderer Art, Ostfildern 2014.

Zulehner P. M., Müller W., Sieberer B. (Hrsg.), Der Reichtum der Kirche sind ihre Menschen. Pfarrgemeinderäte beleben die Kirchengemeinden. Bericht über eine Umfrage, Ostfildern 2010, S. 50-51. 